\title{
Vision-based Experimental Investigation of Two-phase Flow in a rising pipe
}

\author{
Shan $\mathrm{Fu}^{1}$ and Hoi Yeung ${ }^{2}$ \\ ${ }^{1}$ s.fu@cranfield.ac.uk, ${ }^{2}$ h.yeung@cranfield.ac.uk \\ Department of Process and System Engineering, School of Engineering \\ Cranfield University, Cranfield, Bedfordshire, MK43 0AL, UK.
}

Corresponding Author: S. Fu

\begin{abstract}
Multiphase pipeline flow is very important to oil, gas and process industry. This is a class of problems where comprehensive simulations, although desirable on physical grounds, are not practical due to the large aspect ratio between the pipe length and diameter. In order to enhance the understanding of the pipeline flow, as well as to validate and identify the critical aspects of these models, intensive experimental data are needed. This will define how these models can be improved.
\end{abstract}

In this paper, we report our recent work in this aspect. A transparent pipeline, consisting of horizontal and rising sections, was established for conducting the experiments. The purpose of the experiments was to investigate the transition between the basic flow patterns. The flow states were recorded into image sequences using digital camera. Since the required viewing area was much larger than that the required magnification could be satisfied, Image sequence analysis algorithms were developed based on optical flow detection theory to overcome this issue. Apart from the algorithm and experimental results, we also report our further planed research.

Keywords: Two-phase Flow, Pipeline Flow, Image Processing, Optical Flow.

\section{Introduction}

Multiphase pipeline flow is not only very important to oil, gas and process industry but also very complex and scientifically challenging. In the context of the flow in pipeline, the multiphase flows of interest are simulated using one-dimensional transient mathematical models [1][2][3]. This is a class of problems where comprehensive simulations, although desirable on physical grounds, are not practical due to the large aspect ratio between the pipes length and diameter. In order to enhance the understanding of the pipeline flow, as well as to validate and identify the critical aspects of these models, intensive experimental data are needed. This will define how these models can be improved.

Generally, experimental research has been trying to establish a relationship between the flow states and the way that the two phases are mixed. The classification of the flow pattern has been done by visual observation. The standard reference on the pattern classification is Alves [4] who classified the basic flow 
patterns as bubbly, slug, plug, stratified, wavy and annular flow. Manhane et al.[5] presented a useful map indicating the relationship between the flow pattern to the gas and liquid velocities at the inlets. Many similar works can be found and in Taitel's review paper [6] it was concluded that the distinction between the various flow patterns is not always clear. The research of this kind, however, will not reveal the mechanism of the transition of flow patterns during the process. The convection and shape of the deformable interface can be significantly affected by complex factor, e.g. the local continuous phase turbulence [7].

Some researches were carried out to study the local interaction between the liquid and the gas. The flow patterns and the evolution/transitions can be expressed in detail by the state of the flow, which is the combination of the instantaneous shape of the liquid/gas interface and the fluid velocities around the interface. Since the classification of the flow pattern has been done by visual observation, static image and video sequences are popular techniques of recording the patterns. Gopal and Jepson indicated that the image sequence of the flow through a transparent pipe could provide the combined information of the fluid velocity and the void profiles in slug flow [8]. These data can be used to conduct a detailed analysis of the local characteristics within slugs, and also to develop mathematical models to predict the velocity and void profiles in the different regions of the slug. Small bubble and the water/gas interface of larger bubbles were manually extracted and used as the tracking features in their technique of obtaining the velocity information. The use of gas bubbles as the velocity tracking feature is debatable in this case because these are constantly deforming and coalescing due to the difference in velocity of liquid and gas. Even with additional solid tracking particles, the shape of the liquid-gas interface and the liquid velocity distribution can hardly be measured simultaneously due to the significant scale difference between the interface and tracking particles. Having reviewed alternative measuring techniques for two-phase flow, e.g. LDA, tomography and PIV, Polonsky et al presented the results of their experiment [9] in which they tried to establish a relationship between the Taylor bubble motion and the water velocities ahead of it. Even though they used a complicated optical arrangement, i.e. synchronised multiple cameras along the pipe, they can only use the motion of bubble nose to indicate the motion of the bubble, because neither of the cameras can cover the whole length of the bubble whilst a certain magnification needed to be satisfied for PIV measurement to be performed. The velocity distribution, motion and shape of the moving bubble became separated measurements, and therefore the correlation among the three would degrade. Recent work by Saad and Bugg [10] provided PIV measurements of the water velocity distribution around a Taylor bubble rising in stagnant water with an assumption that the Taylor bubble did not change its shape during the period of measurement. In contrast, research reported by Tomiyama et al [11] was concentrated in the rising velocity and shapes of individual bubbles in vertical pipes.

So far, there is no experimental research reported on the evolution of the interface and linked it to the local instantaneous velocity distribution for the two-phase flow in pipeline. We believe that the main reason for the lack of research done in this area has been due to the difficulties in processing and analysis techniques for the images obtained from the experiments. In order for traditional PIV algorithms to be successfully carried out, the magnification of the image needs to be high so that the distribution of the tracking particles can be recognised in each images. The image of this kind, however, will not be able to cover an adequate area of the flow field where the whole process of the interface development takes place. If we want to observe the development of the interface from one viewpoint, the image obtained will not have an adequate resolution for traditional PIV algorithms to perform correctly.

In contrast with traditional feature-based PIV analysis algorithm, Optical Flow algorithms treats image patterns as two dimensional functions instead of a collection of individual image features, and the image motion would be considered as functional deformation, such as translation, rotation and scaling. Based on such a nature of the algorithm, the motion analysis does not require as high resolution as PIV algorithms 
do. OF algorithms, however on the other hand, require to incorporate with the nature of the moving objects[12], especially when the objects are deformable, to get optimised motion detection. To extend OF algorithms for detecting fluid motion will be a great scientific challenge as well as important contribution to both experimental fluid mechanics and computer vision communities.

\section{From Optical Flow to Liquid Flow}

Based on the assumption that the appearance of each constituent element of the flow should keep constant brightness in the image sequence for a certain period of time. The following equation expresses the brightness constant constraint

$$
E(x, y, t)=E(x+u, \quad y+v, \quad t+1)
$$

where $E(x, y, t)$ was the image brightness at the point $(x, y)$ in the image $t$, and the time interval between the images is the unit time period. Two meanings were expressed by this equation; (a) the image point $(x, y)$ at time $t$ and image point $(x+u, y+v)$ at time $t+1$ were corresponding to the same constituent element, (b) the brightness of these two image points should be the same. Using the chain rule for differentiation, this equation could be expanded to give

$$
E_{x} u+E_{y} v+E_{t}=0
$$

where $E_{x}=\partial E / \partial x, E_{y}=\partial E / \partial y, E_{t}=\partial E / \partial t$

We suggested that the velocity distribution of the flow could be extracted by monitoring the movement of the brightness pattern in the image sequence of the pipe flow. Only with the equation (1), however, we can't work out the movement simply because there are two unknowns with the equation. According to the nature of the general fluid flow, we suggested that each element of the flow should travel in the similar direction of its neighboring elements and therefore the directional smoothness constraint [13]. We used the following measurement,

$$
\xi_{b}=E_{x} u+E_{y} v+E_{t}
$$

to measure the smoothness of brightness of every elements,

$$
\xi_{c}^{2}=\left(\frac{\partial(u / v)}{\partial x}\right)^{2}+\left(\frac{\partial(u / v)}{\partial y}\right)^{2}
$$

to measure the smoothness of the element travel direction. The two dimensional movement of every element can be obtained by minimizing the total measurement given by

$$
\xi^{2}=\iint\left(\lambda^{2} \xi_{c}^{2}+\xi_{b}^{2}\right) d x d y
$$


Some results from the technique based on the above theory were presented in previous paper [14]. However, we found the issue of "large displacement between the consecutive images" and "large scale difference between the image of the interface and tracking particles" need to be addressed carefully when we applied this theory in the multi-phase pipeline flow:

The displacement between a pair of consecutive images depended on both the flow speed and the imaging rate that depended not only upon your experiment budget but also the state of art technology. In the experiment, which will be described below, a normal speed $(25 \mathrm{~Hz})$ digital camera was used. The image sequence captured by the camera was good for the water/gas interface description, however was not good for the flow field measurement due to the large inter-frame image displacement. The inherent reason in terms of the algorithm was that the linear chain rule was used to expend the brightness constant constraint. Although the higher order expansion of this constraint was used for other optical flow detection technique [6], a high noise level found in the flow images prevent an adequate second derivative of the image data from being estimated.

\section{Multi-scale modification scheme}

As we have pointed out earlier in this paper that the brightness constant constraint is expressed using linear Taylor expansion, the image movement at each point can only be estimated accurately within its local monotonic (increase/decrease in terms of the image brightness) region. A large scale (high resolution) image should contain more details of the flow field appearance, which means the size of local monotonic region is small. A lower scale image contains fewer of the radical intensity variations associated with changes in local monotonic state. It follows that at lower scale the average size of the spatial region represented by the local monotonic region will increase and, therefore, more accurate estimates of image flow may be expected.

Based on the above discussion, we used the following multi-scale modification procedure:

1. Deliberately shrink each image in the sequence by the same amount (the level of shrinkage should depend upon the expected magnitude of the largest inter-frame changes). The shrinking procedure must be an integrating, re-sampling process; divide each image into regular squares and let the intensity value assigned to each square be the average of the original image intensities over that square. By representing each square as a single pixel, the original images are shrunk to a smaller scale. Each image, therefore, is represented in a series of images at pyramidal scales. This procedure could be thought of as re-imaging each view. A key feature of the reduced scale sequence is that inter-image differences, in terms of brightness pattern movement, become smaller, and the high frequency image component (e.g. fine surface texture) was smoothed away gradually due to regional integration.

2. Work on the reduced scale image sequence to obtain an initial estimate of the inter-frame image change. The estimated image movement $M$ is divided into parts $M_{0}$ and $M$, the initial and fractional estimates.

$$
M_{\mathrm{s}}=\left.M_{0}\right|_{\mathrm{s}}+\left.M^{\prime}\right|_{\mathrm{s}}
$$


$M$ has two axial components $u$ and $v$, which are also divided into two parts,

$$
\left.u\right|_{\mathrm{s}}=\left.u_{0}\right|_{\mathrm{s}}+\left.u^{\prime}\right|_{\mathrm{s}} \text {, and }\left.\quad v\right|_{\mathrm{s}}=\left.v_{0}\right|_{\mathrm{s}}+\left.v^{\prime}\right|_{\mathrm{s}}
$$

The subscript $\mathrm{s}$ denotes the scale at which the computation is performed. At the smallest (lowest, say $\mathrm{s}=\mathrm{N}$ ) scale, let $\left.M_{0}\right|_{\mathrm{N}}=0$.

3. Using the values obtained in the previous step as an initial estimate, work on the image sequence at a larger scale (i.e. on a sequence developed from the same original but shrunk to a lesser degree) to produce an improved estimate of local image flow. The initial estimate used at each scale is the estimate of the whole movement obtained from the previous scale;

$$
\left.M_{0}\right|_{\mathrm{s}-1}=M_{\mathrm{s}} \times f s, \quad \text { where } f s \text { is a scale factor }
$$

4. Repeat step 3 until the process has been applied to the original, full-scale image sequence. This procedure did not only solve the large-disparity issue, but also for the issue caused by the scale difference between the water/gas interface and the tracking particles.

\section{Experiment}

A mock-up of a section oil collection pipeline was constructed, shown below.

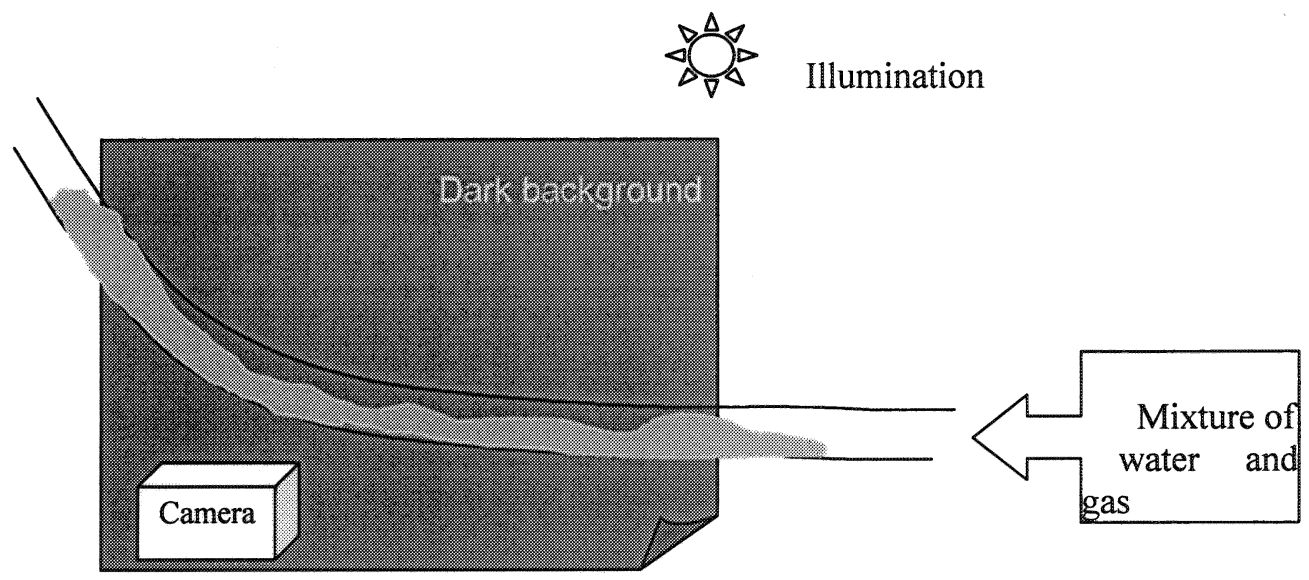

Figure 1. Sketch of the experiment set-up

The camera observed the pipeline against a dark background. The illumination was from the top of the pipe. The internal diameter was $200 \mathrm{~mm}$. The mixture of air and water was input into the pipe from the lower end of the pipe. The water was seeded with tracking particles.

The typical appearance of the recorded image sequence was shown below in the figure 2 . 


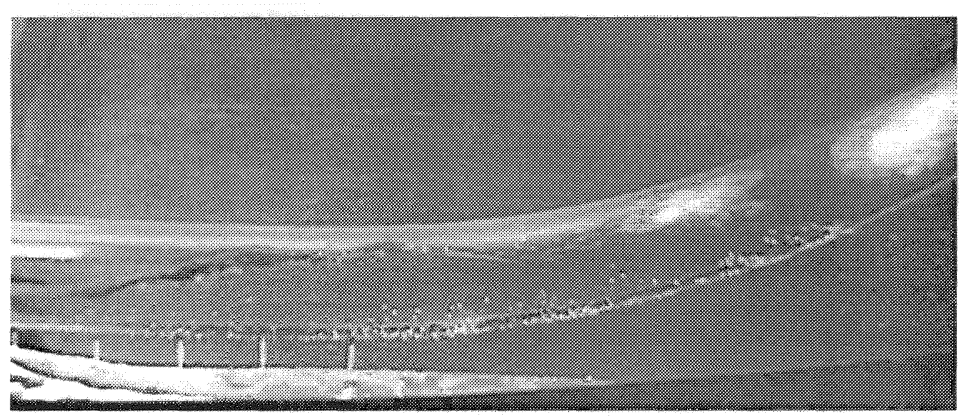

Figure 2. Recorded flow image

\section{Results and Discussion}

We were particularly interested in the slug initiating mechanism and therefore images that were studied so far were mostly the bubbly flow images. One of the examples was shown below.

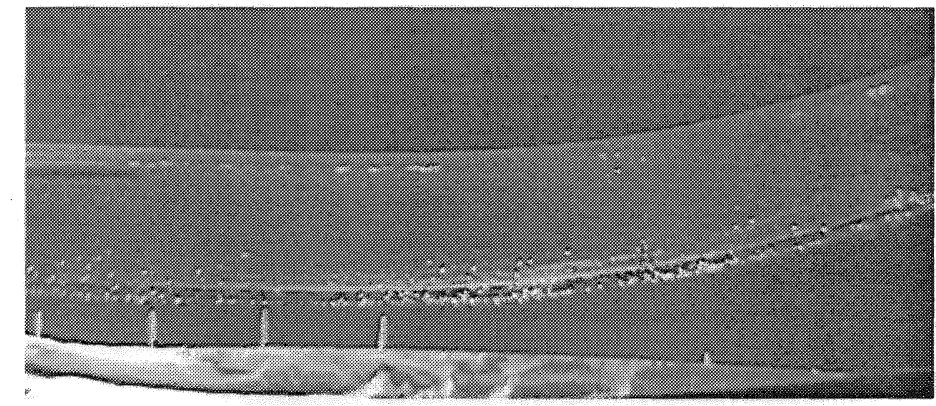

(a) Recorded bubbly flow image

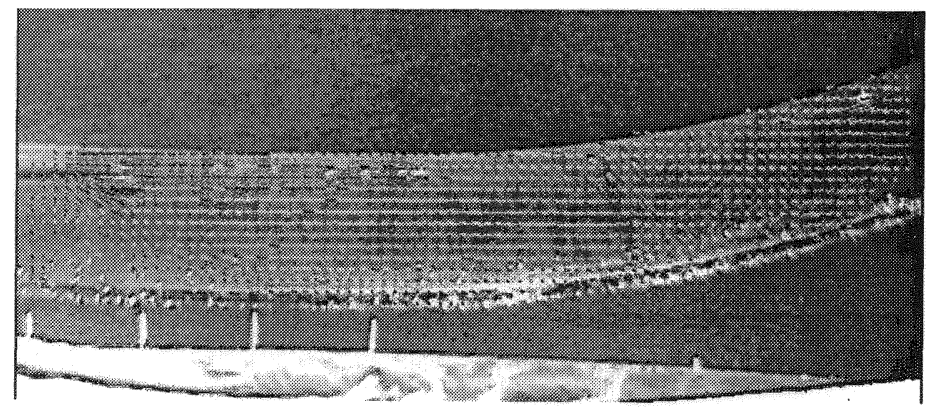

(b) Velocity distribution of water

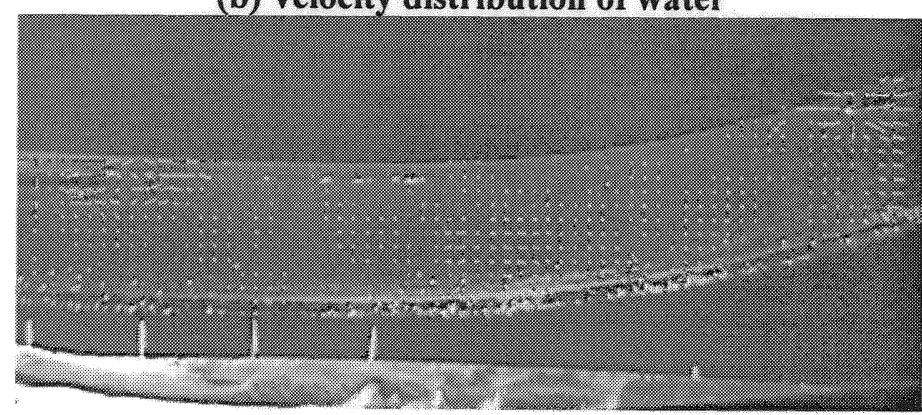

(c) Acceleration distribution of water Figure 3. Bubbly flow image analysis 
Although the tracking particles were visible individually in the images, the algorithm was still capable of extracting the flow velocity information from the sequence. The velocity estimates were accurate enough for the further acceleration calculation, which is indicated clearly in figure 3(a) that the frontier of the water/gas interface travelled faster than its surrounding water and the bubble was expanding while it was travelling.

\section{Conclusion}

We have presented a set of algorithms designed for tracking the image displacement with large scale difference between two types of objects involved in this study, the tracking particles and water/gas interface. The preliminary results presented in this paper demonstrated promising performance of the algorithms. In a pipeline, 3-D characteristics of flow are generally strong and therefore our next stage work will inevitably target the three-dimensional measurement.

\section{Reference:}

[1] Hanich, L. and Thompson, C. Validation of a Novel Algorithm for the Adaptive Calculation of Transient Stratified Flow of Gas, Oil and Water in Pipelines. Int. J. Num. Methods in Engineering, vol. 51(5) pp.579-607, 2001.

[2] Thompson, C. P. and Lezeau, P., Application of a FAS multi-grid method to the simulation of two-dimensional steady incompressible viscous multi-phase flow, Int. J. Num. Methods in Fluids, 28:1217-1239, 1998.

[3] Barnea, D. and Taitel, Y., Stratified Three Phase Flow in Pipes-Stability and Transition, Chem. Eng. Comm. vol. 141-142, pp. 443-460, 1996.

[4] G.E.Alves, Co-current liquid-gas flow in a pipeline contractor, Chem. Process Engineering 50. 1954 pp449-456.

[5] Manhane, J.M., Gregory, G.A. and Aziz, K. A flow pattern map for gas-liquid flow in horizontal pipeline, Int. J. Multiphase Flow, Vol. 1, 1974, pp. 537-553

[6] Taitel, Y., Flow PatternTransition in two phase flow, Proceedings of the Ninth International Heat Transfer Conference, Jerusalem, no. K-14, pp. 237-254

[7] Loth, E., Taeibi-rahni, M. and Tryggvason, G., Deformable Bubbles in a Free Shear Layer, Int. J. Multiphase Flow, Vol. 23, no. 5. 1997, pp. 977-1001

[8] Gopal, M., and Jepson, W. P., Development of Digital Image Analysis Techniques for the Study of Velocity and Void Profiles in Slug Flow. Int. J. Multiphase Flow, Vol. 23, no. 5, 1997, pp. 945-965

[9] S. Polonsky, L. Shemer, D. Barnea, The relation between the Taylor bubble motion and the velocity field ahead of it, Int. J. Multiphase Flow, Vol. 25, no. 6-7. 1999, pp. 957-975

[10]Gaballah A. Saad and James D. Bugg, PIV measurements around a Taylor bubble rising in a stagnant fluid. 4th International Conference on Multiphase Flow, ICMF'2001, New Orleans, LA, U.S.A., May 27-June 1, 2001

[11]Akio Tomiyama, Yusuke Nakahara and Go Morita, Rising velocities and shapes of single bubbles in vertical pipes, 4th International Conference on Multiphase Flow, ICMF'2001, New Orleans, LA, U.S.A., May 27-June 1, 2001

[12]B.K.P. Horn and B.G. Schunk, Determining Optical Flow, Artificial Intelligence, Vol. 17, pp 185-203, 1981

[13]Fu, S. and T. P. Pridmore Image Flow Field Detection, Proc. Third International Conference on Signal Processing (ICSP'96) Vol. 2, pp. 1090-1093, Beijing, Oct. 14-17, 1996

[14]S. Fu and Y. Wu, Detection of Velocity distribution of a Flow Field Using sequences of Schlieren Images, Optical Engineering, vol. 40 no. 8, (2001), pp 1661-1666 